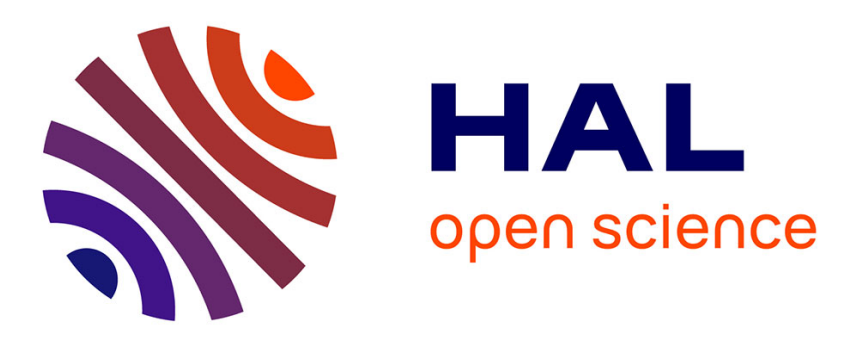

\title{
The revised ghent nosology; reclassifying isolated ectopia lentis
}

\author{
A. Chandra, D. Patel, A. Aragon-Martin, Amélie Pinard, Gwenaëlle \\ Collod-Béroud, C Comeglio, C. Boileau, L. Faivre, D. Charteris, a H Child, et \\ al.
}

\section{To cite this version:}

A. Chandra, D. Patel, A. Aragon-Martin, Amélie Pinard, Gwenaëlle Collod-Béroud, et al.. The revised ghent nosology; reclassifying isolated ectopia lentis. Clinical Genetics, 2015, 87 (3), pp.284287. 10.1111/cge.12358 . hal-01670143

\section{HAL Id: hal-01670143 https://hal.science/hal-01670143}

Submitted on 21 Dec 2017

HAL is a multi-disciplinary open access archive for the deposit and dissemination of scientific research documents, whether they are published or not. The documents may come from teaching and research institutions in France or abroad, or from public or private research centers.
L'archive ouverte pluridisciplinaire HAL, est destinée au dépôt et à la diffusion de documents scientifiques de niveau recherche, publiés ou non, émanant des établissements d'enseignement et de recherche français ou étrangers, des laboratoires publics ou privés. 


\section{The revised ghent nosology; reclassifying isolated ectopia lentis}

Chandra A., Patel D., Aragon-Martin J.A., Pinard A., Collod-Béroud G., Comeglio P., Boileau C., Faivre L., Charteris D., Child A.H., Arno G.

Inherited ectopia lentis (EL) is most commonly caused by Marfan syndrome (MFS), a multisystemic disorder caused by mutations in FBN1. Historically the diagnosis for patients with EL who have no systemic features of MFS is isolated EL (IEL). However, the Ghent nosology for MFS was updated in 2010 and made some important alterations. In particular, patients with EL and a FBN1 mutation are now categorically diagnosed with MFS, if their mutation has previously been described with aortic dilation/dissection. This carries significant systemic implications, as many patients previously diagnosed with IEL are now reclassified. We provide a review of all published cases of IEL caused by FBN1 mutations over the last 20 years to assess what impact the new Ghent nosology has on these. Indeed, 57/123 probands (46.3\%) are now classified as MFS according to the revised Ghent nosology and 37/96 mutations (38.5\%) reported to cause isolated EL have also been found in patients with aortic dilation/dissection. These findings suggest that EL caused by mutations in FBN1 is actually part of a spectrum of fibrillinopathies with MFS, and the term 'IEL' should be avoided in such cases.

\section{Conflict of interest}

All authors declare no conflict of interest.

\author{
A. Chandra ${ }^{a, b, c}$, D. Patel ${ }^{a}$, \\ J.A. Aragon-Martin ${ }^{\mathrm{d}}$, \\ A. Pinard ${ }^{e, f}$, \\ G. Collod-Béroude,f, \\ P. Comeglio ${ }^{d}$, C. Boileau ${ }^{g, h, i, j,}$ \\ L. Faivre ${ }^{k, l}$, D. Charteris ${ }^{a, b}$, \\ A.H. Child ${ }^{d}$ and G. Arno ${ }^{b}$
}

aVitreoretinal Unit, Moorfields Eye Hospital, London, UK, ' Inherited Eye Diseases, UCL Institute of Ophthalmology, London, UK, 'Vitreoretinal unit, Royal Victoria Eye and Ear Hospital, Melbourne, Australia, ${ }^{\mathrm{d} C}$ Cardiac and Vascular Sciences, St Georges University of London, London, UK, ${ }^{e}$ Aix Marseille Université, GMGF, 13385, Marseille, France, ' INSERM, UMR_S 910, 13385, Marseille, France, Institut National de la Santé et de la Recherche Médicale Unité 698, Hôpital Bichat, Paris, France, ${ }^{\mathrm{h}}$ Centre de Référence pour les Syndromes de Marfan et Apparentés, Service de Cardiologie, Hôpital Bichat, Assistance Publique - Hôpitaux de Paris, Paris, France, 'Service de Cardiologie, Hopital Bichat, Assistance Publique, Hôpitaux de Paris, Paris, France, JUnité de Formation et de Recherche de Médecine, Université Paris Diderot, Paris, France, kEquipe GAD, EA 4271, Université de Bourgogne, Dijon, France, and 'Centre de Génétique et Centre de Référence Anomalies du Développement et Syndromes Malformatifs, Hôpital d'Enfants, Dijon, France

Key words: ectopia lentis - FBN1 isolated ectopia lentis - Marfan syndrome - mutation

Corresponding author: Gavin Arno, Inherited Eye Disease, UCL Institute of Ophthalmology, London, UK. Tel: 02076086971. e-mail: g.arno@ucl.ac.uk 
The human crystalline lens is held in its natural position behind the iris by the zonular filaments (ZFs). The ZFs are composed of microfibrils, and the most important macromolecular components of $\mathrm{ZF}$ is fibrillin-1. Ectopia lentis (EL) (subluxation or dislocation of the lens, EL) is because of the abnormal embryogenesis, stretching or fragmentation of ZF (1).

Fibrillin-1, which is the most abundant of the constituent fibrillins is encoded by $F B N 1$, a $237 \mathrm{~kb}$ gene comprising 65 exons located at $15 \mathrm{q} 21.1$. Mutations in FBN1 cause Marfan syndrome (MFS, MIM\#154700) and several related disorders including autosomal dominant ascending aortic aneurysm, familial arachnodactyly, MASS phenotype (Mitral valve prolapse, borderline non-progressive Aortic root enlargement, Skin and Skeletal findings, MIM\#604308), stiff skin syndrome (SSKS, MIM\#184900), Weill-Marchesani syndrome 2 (WMS2, MIM\#608328), acromicric dysplasia (MIM\#102370) and autosomal dominant EL (MIM\#129600). There is significant overlap between these phenotypes, and to aid the accurate description of MFS diagnostic criteria have been established (2, 3) which score the clinical features of MFS. The latest iteration of the Ghent nosology for MFS proposed a more prominent role for molecular genetic testing of $F B N 1$ and recommended more weight be given to the two cardinal features of MFS (aortic root dilatation and EL) (4).

One major change in the 2010 revision to the previous Ghent nosology was in the diagnosis of familial EL (4). Previously this diagnostic category was applied to patients with predominant EL with or without skeletal features in the absence of significant aortic root dilatation in whom a FBN1 mutation was identified. In the revision, the diagnosis of 'isolated EL' is excluded in patients under the age of 20 since cardiovascular development is incomplete before this age. In addition, patients with EL and an FBN1 mutation are now categorically diagnosed with MFS if their mutation has previously been identified with aortic dilation/dissection. This particular change has significant implications, as many patients previously diagnosed with familial or isolated EL are now reclassified as MFS.

The aim of this study was to review all published cases of isolated EL caused by mutations in FBN1 between 1993 and 2013 and assess the impact of the revised Ghent nosology on these diagnoses. We also provide a current list of mutations causing EL which are not associated with aortic dilation/dissection.

\section{Methods}

Papers describing mutations in patients with isolated EL or familial EL were identified from Pubmed between January 1993 and January 2013. Search terms used were: ((()((()("Ectopia Lentis”[Mesh]) OR ectopia lentis[Text Word])) OR "marfan syndrome" [MeSH Major Topic]) OR marfan[Text Word]) AND mutation)) AND fibrillin[Text Word]) AND mutation[Text Word]). The resulting list of 434 publications was manually filtered to 43 describing FBN1 mutations in patients having EL (Table S1, Appendix S1, Supporting Information). Patients were included in this analysis if they were described as having EL without aortic dilation/dissection or a family history of MFS. Patients with a clinical description of incomplete MFS with EL and other systems involved were included if their diagnosis according to the revised Ghent nosology was of EL Syndrome (ELS) and not MFS (i.e. without aortic dilation/dissection or a family history of MFS). This list was completed with unpublished data from Universal Mutation Database (UMD)-FBN1 (5) (2946 mutations in the online updated version).

The mutations were investigated on the UMDFBN1 (5, 6), the public Human Genome Mutation Database (HGMD), National Center for Biotechnology Information (NCBI)/Pubmed, and an internal database of over 300 FBN1 mutations held at the Sonalee Laboratory, St George's University of London. We searched for evidence that these mutations were also reported in cases with aortic dilation/dissection which according to the revised Ghent nosology would result in the reclassification of EL to MFS in each case.

\section{Results}

A database was created comprising 198 patients with EL and a FBN1 mutation who did not fulfil the diagnostic criteria for MFS at the time of publication (Appendix $\mathrm{S} 1$ ). This database represented 96 independent mutations found in 123 probands and 75 family members. Fifty-one of these patients were under the age of 20 at the time of publication and 45 had no accurate age reported. The mean age of the remaining 102 patients was $39.6 \pm 13.7$ years. According to the revised Ghent nosology true isolated EL cannot be diagnosed in patients under 20 (4) immediately suggesting that $51 / 198(25.2 \%)$ of these cases cannot now be classified as isolated EL based on the current guidelines.

This investigation resulted in the reclassification of $57 / 123$ probands $(46.3 \%$ ) from EL to MFS according to the revised Ghent nosology based on a reported association of the mutation with aortic dilation/dissection in another patient. Furthermore, only 42/123 (34.1\%) of probands can be described as isolated EL based on their mutation and age $\geq 20$ years (Table 1 , Fig. 1). In addition, $37 / 96$ mutations $(38.5 \%)$ have been described in patients with aortic dilation/dissection and only 40

Table 1. Reclassification of published isolated EL patients and mutations based on the revised Ghent nosology (4)

\begin{tabular}{lccc}
\hline & Patients & Probands & Mutations \\
\hline Total & 198 & 123 & 96 \\
Excluded: <20 years old & $138(69.7 \%)$ & $81(65.9 \%)$ & 54 \\
$\quad(56.2 \%)$ \\
$\quad$ and/or mutation \\
$\quad$ previously associated \\
$\quad$
\end{tabular}

AD, aortic dilation/dissection; ELS, ectopia lentis syndrome. 


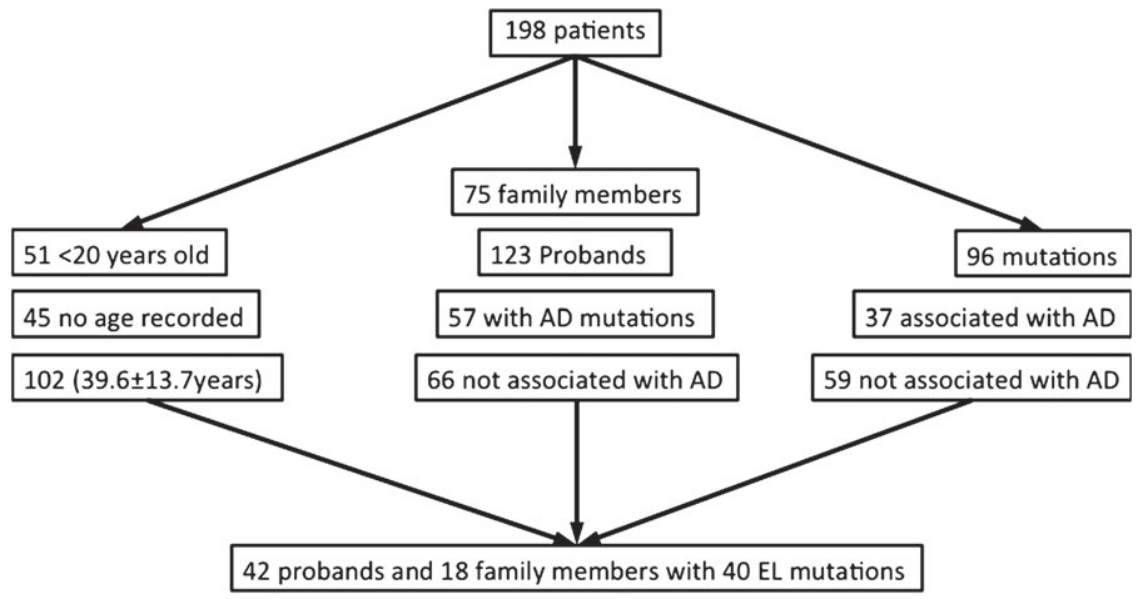

Fig. 1. Flow diagram of reclassification of 198 ectopic-lentis EL patients published between 1993 and 2013 . Forty-two probands and 18 family members remain under the diagnosis of EL based on no association of their mutation with aortic dilation/dissection and age $\geq 20$ years* $(*$ patients with no age data are also included).

(41.7\%) of mutations can be described as EL mutations based on patient age $\geq 20$ years and no association with aortic dilation/dissection (Table 1, Fig. 1).

Fifteen mutations were reported in more than one proband with isolated EL and account for 42/123 probands identified (Appendix S1). Nine of fifteen of these mutations are now reclassified as MFS and include the five most prevalent mutations to be reported in three or more independent probands. The remaining six mutations may be considered EL mutations of $F B N 1$, but this must be interpreted with caution as these reports include only two independent families and include patients under the age of 20 .

\section{Discussion}

In this study we reanalysed the diagnosis of isolated EL in 198 patients published in the last 20 years. This work describes the major changes in the classification of isolated or familial EL as a consequence of the 2010 revision to the Ghent nosology for MFS. Furthermore, $46.3 \%$ of probands previously not fulfilling the Ghent criteria for MFS now do based on the FBN1 mutation they carry.

The findings described here suggest that EL caused by mutations in FBN1 is actually part of a spectrum of fibrillinopathies with MFS rather than a distinct or 'isolated' phenotype. This also shows the importance of having a curated and up to date database of $F B N 1$ mutations known to cause MFS because this is now important information in the diagnosis of new MFS cases.

The clinical implications of a diagnosis of MFS are significant, and careful management of these 'isolated EL' patients, in particular children, is of paramount importance. Regular follow-up including annual cardiovascular imaging is recommended in patients with EL $(4,6,7)$, which may lead to a reclassification to MFS in such individuals. This is essential as even in families with a history of isolated
EL, late onset aortic disease is possible. Counselling of families with seemingly isolated EL should include the possibility of more severe diagnoses in the future or in their offspring. We believe the data presented here in conjunction with the UMD-FBN1 mutation database (5) can be used as a guide when an FBN1 mutation is identified in a patient with seemingly isolated EL and can certainly highlight those mutations identified in patients with seemingly isolated EL that are known to carry a significant risk of aortic dilatation and/or dissection.

Of significant interest is that since the 2010 watershed of the revised Ghent nosology for MFS, patients are still being described as isolated EL with FBN1 mutations already known to cause MFS $(8,9)$. A further point of interest involves those papers that describe patients with MFS, with (to our knowledge) truly novel FBN1 changes and not enough clinical data to fulfil this diagnosis (10).

\section{Conclusion}

It may now be argued that the term FBN1 associated isolated EL should be dismissed. We have shown that of 123 probands originally grouped under this term, 57 are now classified as MFS. For the remaining patients (42 probands and 18 family members aged $\geq 20$ years whose mutation has not been associated with aortic dilation/dissection), Loeys et al. (4) suggested that the phrase ELS be used to illustrate that other systems may also be involved at time of diagnosis or during follow-up. This term is inclusive for the patients who will not develop complications in other systems. Physicians should be aware that some patients will be reclassified as MFS if aortic dilatation appears or if the mutation is subsequently described in association with aortic dilation/dissection. The ELS has been estimated to represent less than $8 \%$ in an adult population (7). The diagnosis of true isolated EL should be reserved for those with autosomal recessive EL and 
to date the only gene shown to cause this condition is ADAMTSL4. There may be however further genetic causes of isolated EL as shown in our own studies $(11,12)$. Such families will require careful genetic and clinical investigations in order to elucidate the genetic mechanisms at work. Probable candidate genes should include those whose protein products interact with fibrillin-1 and/or ADAMTSL4 in the development and function of the $\mathrm{ZF}$.

\section{Supporting Information}

The following Supporting information is available for this article:

Appendix S1. References for Table S1.

Table S1. All FBN1 mutations reported to cause isolated EL published between January 1993 and January 2013.

Additional Supporting information may be found in the online version of this article.

\section{References}

1. Greene VB, Stoetzel C, Pelletier V et al. Confirmation of ADAMTSL4 mutations for autosomal recessive isolated bilateral ectopia lentis. Ophthalmic Genet 2010: 31: 47-51.

2. Beighton P, de Paepe A, Danks D et al. International nosology of heritable disorders of connective tissue, Berlin, 1986. Am J Med Genet 1988: 29: $581-594$.
3. De Paepe A, Devereux RB, Dietz HC, Hennekam RC, Pyeritz RE. Revised diagnostic criteria for the Marfan syndrome. Am J Med Genet 1996: 62: 417-426.

4. Loeys BL, Dietz HC, Braverman AC et al. The revised Ghent nosology for the Marfan syndrome. J Med Genet 2010: 47: 476-485.

5. Collod-Beroud G, Le Bourdelles S, Ades L et al. Update of the UMDFBN1 mutation database and creation of an FBN1 polymorphism database. Hum Mutat 2003: 22: 199-208.

6. Faivre L, Collod-Beroud G, Callewaert B et al. Pathogenic FBN1 mutations in 146 adults not meeting clinical diagnostic criteria for Marfan syndrome: further delineation of type 1 fibrillinopathies and focus on patients with an isolated major criterion. Am J Med Genet A 2009: 149A: 854-860.

7. Faivre L, Collod-Beroud G, Ades L et al. The new Ghent criteria for Marfan syndrome: what do they change? Clin Genet 2012: 81: $433-442$.

8. Li H, Qu W, Meng B et al. Identification and study of a FBN1 gene mutation in a Chinese family with ectopia lentis. Mol Vis 2012: 18: 504-511.

9. Yang G, Chu M, Zhai X, Zhao J. A novel FBN1 mutation in a Chinese family with isolated ectopia lentis. Mol Vis 2012: 18: 945-950.

10. Micheal S, Khan MI, Akhtar F et al. Identification of a novel FBN1 gene mutation in a large Pakistani family with Marfan syndrome. Mol Vis 2012: 18: 1918-1926.

11. Aragon-Martin JA, Ahnood D, Charteris DG et al. Role of ADAMTSL4 mutations in FBN1 mutation-negative ectopia lentis patients. Hum Mutat 2010: 31: E1622-E1631.

12. Chandra A, Aragon-Martin JA, Hughes K et al. A genotype-phenotype comparison of ADAMTSL4 and FBN1 in isolated ectopia lentis. Invest Ophthalmol Vis Sci 2012: 53: 4889-4896. 